\title{
Theoretical derivation of Darcy's law for fluid flow in thin porous media
}

\author{
Francisco Javier SUÁREZ-GRAU*
}

\begin{abstract}
In this paper we study stationary incompressible Newtonian fluid flow in a thin porous media. The media under consideration is a bounded perforated $3 D$ domain confined between two parallel plates. The description of the domain includes two small parameters: $\varepsilon$ representing the distance between pates and $a_{\varepsilon}$ connected to the microstructure of the domain such that $a_{\varepsilon} \ll \varepsilon$. We consider the classical setting of perforated media, i.e. $a_{\varepsilon}$-periodically distributed solid (not connected) obstacles of size $a_{\varepsilon}$. The goal of this paper is to introduce a version of the unfolding method, depending on both parameters $\varepsilon$ and $a_{\varepsilon}$, and then to derive the corresponding $2 D$ Darcy's law.
\end{abstract}

AMS classification numbers: 76A20, 76M50, 35B27, 35Q30.

Keywords: Homogenization, Stokes equations, Darcy's law, thin porous media, thin film fluids.

\section{Introduction}

The problem of Stokes fluid flows in a periodically perforated domains with obstacles of the same size as the period has been widely treated in the literature. As is well known, such kind of flows are generally modelled by Darcy's law, see Darcy [8]. From the mathematical point of view, the transition between Stokes equations to Darcy's law was formally obtained in Sanchez-Palencia [12] and rigorously in Tartar [13. Since then, several approaches to derive Darcy's law have been used by classical authors in homogenization theory, such as Allaire [1, Hornung [10] and Lions [11] among others. The goal of this paper is to generalize classical results of perforated media to the case of thin porous media, which by definition includes two small parameters: one called $\varepsilon$ is connected to the fluid film thickness and the other denoted by $a_{\varepsilon}$ to the microstructure representing the size of the obstacles and the interspatial distance between them.

The case of thin porous media has been recently considered taking as microstructure a periodic array of vertical cylinders of size and period $a_{\varepsilon}$ confined between two parallel plates with distance $\varepsilon$ in Fabricius et al. 9]. Thus, depending on the relation between parameters $\varepsilon$ and $a_{\varepsilon}$, different regimes are obtained: proportionally thin porous media $\left(a_{\varepsilon} \approx \varepsilon\right)$, homogeneously thin porous media $\left(a_{\varepsilon} \ll \varepsilon\right)$ and very thin porous medium $\left(a_{\varepsilon} \gg \varepsilon\right)$. For each case, a permeability tensor is obtained by solving local problems. In the critical case, the local problems are $3 \mathrm{D}$, while they are 2D in the other cases, which is a considerable simplification. This result is proved in Fabricius et al. [9] by using the multiscale expansion method, which is a formal but powerful tool to analyze homogenization problems, and later rigorously developed in Anguiano and Suárez-Grau [4 by using an adaptation of the periodic unfolding method, see Arbogast et al. [2] and Cioranescu et al. [6, 7, which is introduced in Anguiano and Suárez-Grau [3].

The goal of this paper is to consider the classical microstructure of perforated domains, i.e. consider $a_{\varepsilon^{-}}$ periodically distributed solid (not connected) obstacles of the same size and then, to study the influence of the thickness of the domain $\varepsilon$ to derive the corresponding Darcy's law. In this case, the restriction $a_{\varepsilon} \ll \varepsilon$ has to be naturally imposed. To our knowledge, this problem has only been considered in the case of modelling a thin film passing a thin porous media by Bayada [5, but only the $2 \mathrm{D}$ case is considered. In this paper, we consider

*Facultad de Matemáticas. Universidad de Sevilla. 41012-Sevilla (Spain) grau@us.es 
the $3 \mathrm{D}$ case and prove the convergence of the homogenization process when $\varepsilon$ and $a_{\varepsilon}$ go to zero. To to this, the microgeometry of the thin porous media requires a version of the unfolding method depending on both parameters $\varepsilon$ and $a_{\varepsilon}$, which can be applied to other problems and also in the $2 \mathrm{D}$ case. As a result, we rigorously derive the corresponding 2D Darcy's law, which is different compared to that obtained in the case of homogeneously thin porous media obtained in Fabricius et al. [9].

The structure of the paper is as follows. In Section 2 we introduce the domain and some useful notation is given in Section 3. In Section 4, we describe the statement of the problem and give the main result (Theorem 4.1), whose proof is provided in Section 5 .

\section{Definition of the domain}

Let $\omega$ be a smooth, bounded and connected set in $\mathbb{R}^{2}$. We consider a positive parameter $\varepsilon$ which describes the height of the domain, i.e. we define $Q_{\varepsilon}=\omega \times(0, \varepsilon)$ as the thin domain without microstructure. To describe the microstructure of the domain $Q_{\varepsilon}$, we consider the positive and small parameter $a_{\varepsilon}$ such that

$$
\lim _{\varepsilon \rightarrow 0} \frac{a_{\varepsilon}}{\varepsilon}=0
$$

We denote $Y=(-1 / 2,1 / 2)^{3}$ the unitary cube in $\mathbb{R}^{3}$ as the reference cell and $T$ an open connected subset of $Y$ with a smooth boundary $\partial T$ such that $\bar{T} \subset Y$. We denote $Y_{f}=Y \backslash \bar{T}$. Thus, for $k \in \mathbb{Z}^{3}$, each cell $Y_{k, a_{\varepsilon}}=a_{\varepsilon} k+a_{\varepsilon} Y$ is similar to the unit cell $Y$ rescaled to size $a_{\varepsilon}$ and $T_{k, a_{\varepsilon}}=a_{\varepsilon} k+a_{\varepsilon} T$ is similar to $T$ rescaled to size $a_{\varepsilon}$. We denote $Y_{f_{k}, a_{\varepsilon}}=Y_{k, a_{\varepsilon}} \backslash \bar{T}_{k, a_{\varepsilon}}$ (see Figure 1). We denote by $\tau\left(\bar{T}_{k, a_{\varepsilon}}\right)$ the set of all translated images of $\bar{T}_{k, a_{\varepsilon}}$. The set
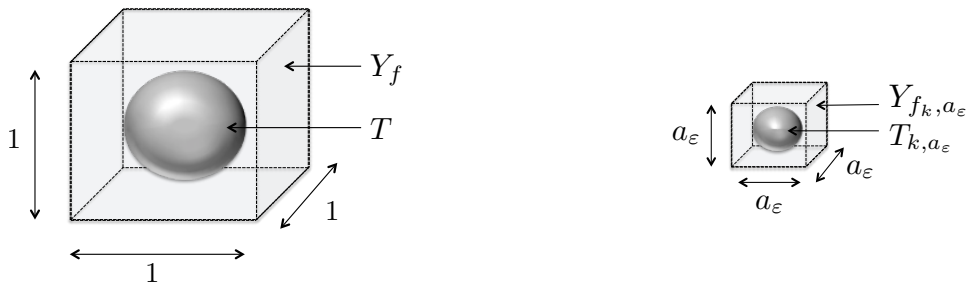

Figure 1: View of the reference cell $Y$ (left) and the rescaled cell $Y_{k, a_{\varepsilon}}$ (right).

$\tau\left(\bar{T}_{k, a_{\varepsilon}}\right)$ represents the obstacles in $\mathbb{R}^{3}$. The thin porous media $\Omega_{\varepsilon}$ is defined by (see Figure 2 )

$$
\Omega_{\varepsilon}=Q_{\varepsilon} \backslash \bigcup_{k \in \mathcal{K}_{\varepsilon}} \bar{T}_{k, a_{\varepsilon}}
$$

where $\mathcal{K}_{\varepsilon}:=\left\{k \in \mathbb{Z}^{N}: Y_{k, a_{\varepsilon}} \cap Q_{\varepsilon} \neq \emptyset\right\}$. By construction, $\Omega_{\varepsilon}$ is a periodically perforated domain with obstacles of the same size as the period. We make the assumption that the obstacles $\tau\left(\bar{T}_{k, a_{\varepsilon}}\right)$ do no intersect the boundary $\partial Q_{\varepsilon}$. We denote by $T_{\varepsilon}$ the set of all the obstacles contained in $\Omega_{\varepsilon}$. Then, $T_{\varepsilon}$ is a finite union of obstacles, i.e.

$$
T_{\varepsilon}=\bigcup_{k \in \mathcal{K}_{\varepsilon}} \bar{T}_{k, a_{\varepsilon}}
$$

As usual when we deal with thin domains, we will use the dilatation in the variable $x_{3}$ given by

$$
z_{3}=\frac{x_{3}}{\varepsilon}
$$

Then, we define the rescaled porous media $\widetilde{\Omega}_{\varepsilon}$ by (see Figure 3 )

$$
\widetilde{\Omega}_{\varepsilon}=\left\{\left(x^{\prime}, z_{3}\right) \in \mathbb{R}^{3}:\left(x^{\prime}, \varepsilon z_{3}\right) \in \Omega_{\varepsilon}\right\} .
$$



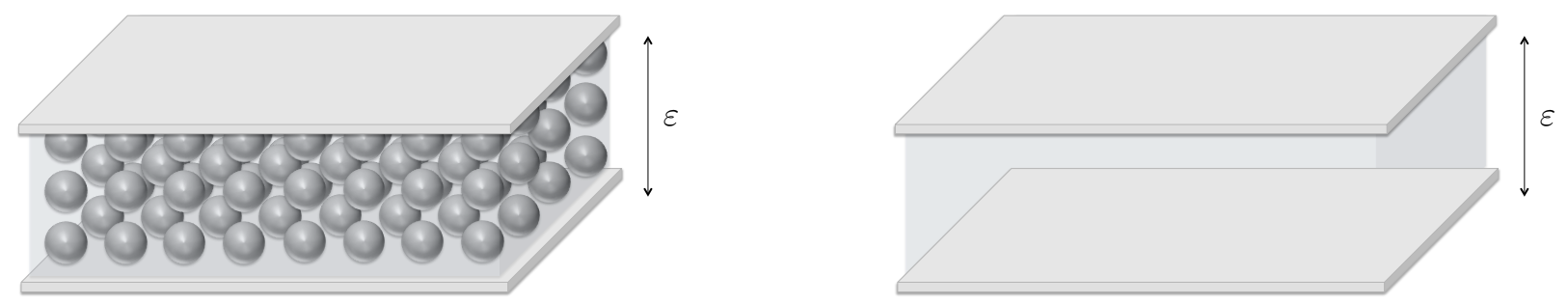

Figure 2: View of the domain $\Omega_{\varepsilon}$ (left) and $Q_{\varepsilon}$ (right).

We also introduce the rescaled sets $\widetilde{Y}_{k, a_{\varepsilon}}$ by (see Figure 3 )

$$
\tilde{Y}_{k, a_{\varepsilon}}=\left\{\left(x^{\prime}, z_{3}\right) \in \mathbb{R}^{3}:\left(x^{\prime}, \varepsilon z_{3}\right) \in Y_{k, a_{\varepsilon}}\right\}
$$

and, in the same way, we define the rescaled fluid part $\widetilde{Y}_{f_{k}, a_{\varepsilon}}$, the rescaled solid part $\widetilde{T}_{k, a_{\varepsilon}}$ of $\widetilde{Y}_{k, a_{\varepsilon}}$ and the union of rescaled obstacles $\widetilde{T}_{\varepsilon}$. Finally, by $\Omega$ we denote the domain with fixed height without microstructure

$$
\Omega=\omega \times(0,1)
$$
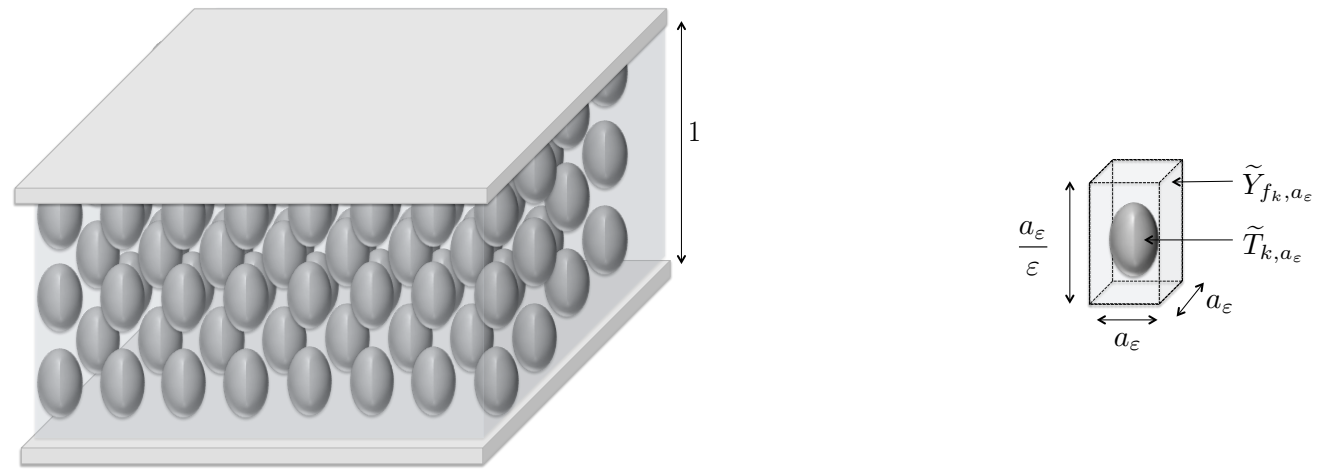

Figure 3: View of the rescaled domain $\widetilde{\Omega}_{\varepsilon}$ (left) and the rescaled cell $\widetilde{Y}_{k, a_{\varepsilon}}$ (right).

\section{Some notation}

Along this paper, the points $x \in \mathbb{R}^{3}$ will be decomposed as $x=\left(x^{\prime}, x_{3}\right)$ with $x^{\prime} \in \mathbb{R}^{2}, x_{3} \in \mathbb{R}$. We also use the notation $x^{\prime}$ to denote a generic vector of $\mathbb{R}^{2}$.

In order to apply the version of the unfolding method, we need the following notation: for $k \in \mathbb{Z}^{3}$, we define $\kappa: \mathbb{R}^{3} \rightarrow \mathbb{Z}^{3}$ by

$$
\kappa(x)=k \Longleftrightarrow x \in Y_{k, 1}
$$

Remark that $\kappa$ is well defined up to a set of zero measure in $\mathbb{R}^{3}$, which is given by $\cup_{k \in \mathbb{R}^{3}} \partial Y_{k, 1}$. Moreover, for every $\varepsilon, a_{\varepsilon}>0$, we have

$$
\kappa\left(\frac{x}{a_{\varepsilon}}\right)=k \Longleftrightarrow x \in Y_{k, a_{\varepsilon}} \quad \text { which is equivalent to } \quad \kappa\left(\frac{x^{\prime}}{a_{\varepsilon}}, \frac{\varepsilon z_{\varepsilon}}{a_{\varepsilon}}\right)=k \Longleftrightarrow\left(x^{\prime}, z_{3}\right) \in \widetilde{Y}_{k, a_{\varepsilon}} .
$$


For a vectorial function $\varphi=\left(\varphi^{\prime}, \varphi_{3}\right)$ and a scalar function $\psi$, we introduce the operators $D_{\varepsilon}, \nabla_{\varepsilon}$ and $\operatorname{div}_{\varepsilon}$ by

$$
\begin{gathered}
\left(D_{\varepsilon} \varphi\right)_{i j}=\partial_{x_{j}} \varphi_{i} \text { for } i=1,2,3, j=1,2, \quad\left(D_{\varepsilon} \varphi\right)_{i, 3}=\varepsilon^{-1} \partial_{y_{3}} \varphi_{i} \text { for } i=1,2,3, \\
\nabla_{\varepsilon} \psi=\left(\nabla_{x^{\prime}} \psi, \varepsilon^{-1} \partial_{y_{3}} \psi\right)^{t}, \quad \operatorname{div}_{\varepsilon} \varphi=\operatorname{div}_{x^{\prime}} \varphi^{\prime}+\varepsilon^{-1} \partial_{y_{3}} \varphi_{3} .
\end{gathered}
$$

We denote by $L_{\text {per }}^{2}(Y), H_{\text {per }}^{1}(Y)$, the functional spaces

$$
\begin{aligned}
& L_{\mathrm{per}}^{2}(Y)=\left\{\varphi \in L_{\mathrm{loc}}^{2}\left(\mathbb{R}^{3}\right): \int_{Y}|\varphi|^{2}<+\infty, \quad v(y+k)=v(y) \quad \forall k \in \mathbb{Z}^{3}, \text { a.e. } y \in Y\right\}, \\
& H_{\mathrm{per}}^{1}(Y)=\left\{\varphi \in H_{\mathrm{loc}}^{1}\left(\mathbb{R}^{3}\right) \cap L_{\mathrm{per}}^{2}(Y): \nabla \varphi \in L_{\mathrm{per}}^{2}(Y)^{3}\right\} .
\end{aligned}
$$

We denote by : the full contraction of two matrices, i.e. for $A=\left(a_{i j}\right)_{1 \leq i, j \leq 3}$ and $B=\left(a_{i j}\right)_{1 \leq i, j \leq 3}$, we have $A: B=\sum_{i, j=1}^{3} a_{i j} b_{i j}$.

The canonical basis in $\mathbb{R}^{3}$ is denoted by $\left\{e^{\prime}, e_{3}\right\}$ where $e^{\prime}=\left\{e_{1}, e_{2}\right\}$.

Finally, we denote by $O_{\varepsilon}$ a generic real sequence, which tends to zero with $\varepsilon$ and can change from line to line, and by $C$ a generic positive constant which also can change from line to line.

\section{Statement of the problem and main result}

We consider the following Stokes system in $\Omega_{\varepsilon}$, with homogeneous boundary conditions in the boundary of the obstacles $\partial T_{\varepsilon}$ and the exterior boundary $\partial Q_{\varepsilon}$,

$$
\left\{\begin{aligned}
-\nu \operatorname{div}\left(D u_{\varepsilon}\right)+\nabla p_{\varepsilon}=f_{\varepsilon} & \text { in } \quad \Omega_{\varepsilon}, \\
\operatorname{div} u_{\varepsilon}=0 & \text { in } \quad \Omega_{\varepsilon}, \\
u_{\varepsilon}=0 & \text { on } \partial T_{\varepsilon} \cup \partial Q_{\varepsilon} .
\end{aligned}\right.
$$

In the above system, velocity filed $u_{\varepsilon}$ and (scalar) pressure $p_{\varepsilon}$ are unknown, while $f_{\varepsilon}$ represents the body force and the viscosity is denoted by $\nu$.

To study the asymptotic behavior of the solution we will use the equivalent variational formulation of (4.6) which is the following one: find $u_{\varepsilon} \in H_{0}^{1}\left(\Omega_{\varepsilon}\right)^{3}$ and $p_{\varepsilon} \in L_{0}^{2}\left(\Omega_{\varepsilon}\right)$ such that

$$
\nu \int_{\Omega_{\varepsilon}} D u_{\varepsilon}: D \varphi d x-\int_{\Omega_{\varepsilon}} p_{\varepsilon} \operatorname{div} \varphi d x=\int_{\Omega_{\varepsilon}} f_{\varepsilon} \cdot \varphi d x, \quad \forall \varphi \in H_{0}^{1}\left(\Omega_{\varepsilon}\right)^{3} .
$$

Assuming external body force $f_{\varepsilon} \in L^{2}\left(\Omega_{\varepsilon}\right)^{3}$, it is well known that (4.6) has a unique weak solution $\left(u_{\varepsilon}, p_{\varepsilon}\right) \in$ $H_{0}^{1}\left(\Omega_{\varepsilon}\right)^{3} \times L_{0}^{2}\left(\Omega_{\varepsilon}\right)$, see e.g [14].

Our aim is to describe the asymptotic behavior of the velocity $u_{\varepsilon}$ and the pressure $p_{\varepsilon}$ of the fluid as $\varepsilon$ tends to zero and identify homogenized models coupling the effects of the thickness and its microgeometry. For this purpose, as usual when we deal with thin domains, we use the dilatation $(2.3)$ in order to have the functions defined in the open set with fixed height $\widetilde{\Omega}_{\varepsilon}$ defined in 2.4. Namely, we define $\tilde{u}_{\varepsilon} \in H_{0}^{1}\left(\widetilde{\Omega}_{\varepsilon}\right)^{3}$ and $\tilde{p}_{\varepsilon} \in L_{0}^{2}\left(\widetilde{\Omega}_{\varepsilon}\right)$ by

$$
\tilde{u}_{\varepsilon}\left(x^{\prime}, z_{3}\right)=u_{\varepsilon}\left(x^{\prime}, \varepsilon z_{3}\right), \quad \tilde{p}_{\varepsilon}\left(x^{\prime}, z_{3}\right)=p_{\varepsilon}\left(x^{\prime}, \varepsilon z_{3}\right), \quad \text { a.e. }\left(x^{\prime}, z_{3}\right) \in \widetilde{\Omega}_{\varepsilon} .
$$

Using the transformation (2.3) the rescaled system (4.6) can be rewritten as

$$
\left\{\begin{aligned}
-\nu \operatorname{div}_{\varepsilon}\left(D_{\varepsilon} \tilde{u}_{\varepsilon}\right)+\nabla_{\varepsilon} \tilde{p}_{\varepsilon}=\tilde{f}_{\varepsilon} & \text { in } \quad \widetilde{\Omega}_{\varepsilon} \\
\operatorname{div}_{\varepsilon} \tilde{u}_{\varepsilon}=0 & \text { in } \quad \widetilde{\Omega}_{\varepsilon} \\
\tilde{u}_{\varepsilon}=0 & \text { on } \quad \partial \widetilde{T}_{\varepsilon} \cup \widetilde{\Omega}
\end{aligned}\right.
$$


where $\tilde{f}_{\varepsilon}$ is defined similarly as in 4.8 . Moreover, taking in 4.7 as test function $\tilde{\varphi}\left(x^{\prime}, x_{3} / \varepsilon\right)$ with $\tilde{\varphi} \in H_{0}^{1}\left(\widetilde{\Omega}_{\varepsilon}\right)^{3}$, applying the change of variables (2.3), the variational formulation for the rescaled system $(4.9)$ is then to find $\tilde{u}_{\varepsilon} \in H_{0}^{1}\left(\widetilde{\Omega}_{\varepsilon}\right)^{3}$ and $\tilde{p}_{\varepsilon} \in L_{0}^{2}\left(\widetilde{\Omega}_{\varepsilon}\right)$ such that

$$
\nu \int_{\widetilde{\Omega}_{\varepsilon}} D_{\varepsilon} \tilde{u}_{\varepsilon}: D_{\varepsilon} \tilde{\varphi} d x^{\prime} d y_{3}-\int_{\widetilde{\Omega}_{\varepsilon}} \tilde{p}_{\varepsilon} \operatorname{div}_{\varepsilon} \tilde{\varphi} d x^{\prime} d y_{3}=\int_{\widetilde{\Omega}_{\varepsilon}} \tilde{f}_{\varepsilon} \cdot \tilde{\varphi} d x^{\prime} d y_{3}, \quad \forall \varphi \in H_{0}^{1}\left(\widetilde{\Omega}_{\varepsilon}\right)^{3} .
$$

We point out that due to the thickness of the domain, it is usual to assume that the vertical component of the external force can be neglected. Thus, let us apply to the fluid an external body force defined by

$$
f_{\varepsilon}=\left(f^{\prime}\left(x^{\prime}\right), 0\right) \quad \text { with } f^{\prime} \in L^{2}(\omega)^{2} .
$$

Our goal then becomes describing the asymptotic behavior of this new sequences $\tilde{u}_{\varepsilon}$ and $\tilde{p}_{\varepsilon}$ when $\varepsilon$ tends to zero. However, the sequence of solutions $\left(\tilde{u}_{\varepsilon}, \tilde{p}_{\varepsilon}\right) \in H_{0}^{1}\left(\widetilde{\Omega}_{\varepsilon}\right)^{3} \times L_{0}^{2}\left(\widetilde{\Omega}_{\varepsilon}\right)$ is not defined in a fixed domain independent of $\varepsilon$ and $a_{\varepsilon}$ but rather in a varying set $\widetilde{\Omega}_{\varepsilon}$. Thus, in order to pass to the limit if $\varepsilon$ tends to zero, convergences in fixed Sovolev spaces (defined in $\Omega$ ) are used which requires first that $\left(\tilde{u}_{\varepsilon}, \tilde{p}_{\varepsilon}\right)$ be extended to the whole domain $\Omega$. Then, by definition, an extension $\left(\tilde{U}_{\varepsilon}, \tilde{P}_{\varepsilon}\right) \in H_{0}^{1}(\Omega)^{3} \times L_{0}^{2}(\Omega)$ of $\left(\tilde{u}_{\varepsilon}, \tilde{p}_{\varepsilon}\right)$ is defined on $\Omega$ and coincides with $\left(\tilde{u}_{\varepsilon}, \tilde{p}_{\varepsilon}\right)$ on $\widetilde{\Omega}_{\varepsilon}$.

Our main result is referred to the asymptotic behavior of the solution of 4.9$]$ and is given by the following theorem.

Theorem 4.1. Let $\left(\tilde{u}_{\varepsilon}, \tilde{p}_{\varepsilon}\right)$ be the unique solution of problem (4.9). Then the sequence of the extension $\left(a_{\varepsilon}^{-2} \tilde{U}_{\varepsilon}, \tilde{P}_{\varepsilon}\right)$ converges weakly to $(u, p)$ in $L^{2}(\Omega)^{3} \times L^{2}(\Omega)$ with $u_{3}=0$. Moreover, defining $U\left(x^{\prime}\right)=\int_{0}^{1} u\left(x^{\prime}, z_{3}\right) d z_{3}$, it holds

$$
U^{\prime}\left(x^{\prime}\right)=K\left(f^{\prime}\left(x^{\prime}\right)-\nabla_{x^{\prime}} p\left(x^{\prime}\right)\right), \quad U_{3}\left(x^{\prime}\right)=0 \quad \text { in } \omega,
$$

and $p \in H^{1}(\omega) \cap L_{0}^{2}(\omega)$ is the unique solution of the $2 D$ Darcy equation

$$
\left\{\begin{aligned}
\operatorname{div}_{x^{\prime}}\left(K\left(f^{\prime}\left(x^{\prime}\right)-\nabla_{x^{\prime}} p\left(x^{\prime}\right)\right)\right) & =0 \quad \text { in } \omega, \\
\left(K\left(f^{\prime}\left(x^{\prime}\right)-\nabla_{x^{\prime}} p\left(x^{\prime}\right)\right)\right) \cdot n & =0 \quad \text { on } \partial \omega .
\end{aligned}\right.
$$

Here $K \in \mathbb{R}^{2 \times 2}$ is a definite positive matrix with coefficients

$$
K_{i j}=\nu \int_{Y_{f}} D w^{i}(y): D w^{j}(y) d y, \quad i, j=1,2,
$$

where $w^{i}, i=1,2$, is the unique solution of the local Stokes problem

$$
\left\{\begin{aligned}
-\nu \Delta_{y} w^{i}+\nabla_{y} \pi^{i}=e_{i} & \text { in } Y_{f} \\
\operatorname{div}_{y} w^{i}=0 & \text { in } Y_{f} \\
w^{i}=0 & \text { in } T \\
w^{i} \in H_{\mathrm{per}}^{1}\left(Y_{f}\right)^{3}, & \pi^{i} \in L_{\mathrm{per}}^{2}\left(Y_{f}\right) / \mathbb{R} .
\end{aligned}\right.
$$

Remark 4.2. As stated in the Introduction, the derivation of Darcy's law flows in thin porous media has been studied in [4, 9] where the microstructure is a periodic array of vertical cylinders of size and period $a_{\varepsilon}$ and thickness $\varepsilon$. As a result, three different regimes depending on the relation of parameters $\varepsilon$ and $a_{\varepsilon}$ are obtained. The relation considered in this paper, $a_{\varepsilon} \ll \varepsilon$, can be framed in the regime called homogeneously thin porous media. Although the current study and the previous ones allow to derive Darcy's law (4.13) with permeability tensor $K$ obtained by solving local problems, the main difference between them lies in the local problems (4.14), because the particular structure of the cylinders in the case of homogeneously thin porous media allows local problems to be reduced to problems defined in a $2 D$ reference cell while they are defined in $3 D$ in this case. 


\section{Proofs}

In this section we provide the proof of the main result (Theorem 4.1). To to this, we define of the extension of the solution and we establish some a priori estimates in Subsection 5.1. In Subsection 5.2 we introduce the version of the unfolding method depending on both parameters $\varepsilon$ and $a_{\varepsilon}$. A compactness result, which is the main key when we will pass to the limit later, is addressed in Subsection 5.3. Finally, the proof of the Theorem 4.1 is given in Subsection 5.4

\section{$5.1 \quad$ A priori estimates}

In this subsection, we establish sharp a priori estimates of the dilated solution in $\widetilde{\Omega}_{\varepsilon}$. To do this, we first need the Poincaré inequality in thin porous media (for the classical version see [13]).

Lemma 5.1. There exists a positive constant $C$, independent of $\varepsilon$, such that

$$
\|\varphi\|_{L^{2}\left(\Omega_{\varepsilon}\right)^{3}} \leq C a_{\varepsilon}\|D \varphi\|_{L^{2}\left(\Omega_{\varepsilon}\right)^{3 \times 3}}, \quad \forall \varphi \in H_{0}^{1}\left(\Omega_{\varepsilon}\right)^{3} .
$$

Moreover, considering $\tilde{\varphi} \in H_{0}^{1}\left(\widetilde{\Omega}_{\varepsilon}\right)^{3}$ such that $\tilde{\varphi}\left(x^{\prime}, z_{3}\right)=\varphi\left(x^{\prime}, \varepsilon z_{3}\right)$, then it holds

$$
\|\tilde{\varphi}\|_{L^{2}\left(\widetilde{\Omega}_{\varepsilon}\right)^{3}} \leq C a_{\varepsilon}\left\|D_{\varepsilon} \tilde{\varphi}\right\|_{L^{2}\left(\widetilde{\Omega}_{\varepsilon}\right)^{3 \times 3}}
$$

Proof. We observe that $\Omega_{\varepsilon}$ can be divided in small cubes of lateral and vertical length $a_{\varepsilon}$. We consider the periodic cell $Y_{f}$ and have a Friedrichs inequality

$$
\int_{Y_{f}}|\varphi|^{2} d z \leq C \int_{Y_{f}}|D \varphi|^{2} d z
$$

for every $\varphi \in H^{1}\left(Y_{f}\right)^{3}$ such that $\varphi=0$ on $\partial T$, where where the constant $C$ depends only on $Y_{f}$. Then, for every $k \in \mathbb{R}^{3}$, by the change of variable

$$
k+z=\frac{x}{a_{\varepsilon}}, \quad d z=\frac{d x}{a_{\varepsilon}^{3}}, \quad \partial_{z}=a_{\varepsilon} \partial_{x},
$$

we rescale 5.17) from $Y_{f}$ to $Y_{f_{k}, a_{\varepsilon}}$. This yields that, for every function $\varphi(x) \in H^{1}\left(Y_{f_{k}, a_{\varepsilon}}\right)^{3}$, one has

$$
\int_{Y_{f_{k}, a_{\varepsilon}}}|\varphi|^{2} d x \leq C \int_{Y_{f_{k}, a_{\varepsilon}}}|D \varphi|^{2} d x,
$$

with the same constant $C$ as in (5.17). Summing previous inequality for every $k \in \mathcal{K}_{\varepsilon}$ and gives (5.15).

In fact, we must consider separately the periods containing a portion of $\partial Q_{\varepsilon}$, but they yield at a distance $O\left(a_{\varepsilon}\right)$ of $\partial Q_{\varepsilon}$, where $\varphi$ is zero, and then the corresponding inequality is immediately obtained.

Finally, by taking in $(5.15)$ the change of variables 2.3$)$, we get 5.16 .

We give a priori estimates for velocity $\tilde{u}_{\varepsilon}$ in $\widetilde{\Omega}_{\varepsilon}$.

Lemma 5.2. There exists a positive constant $C$, independent of $\varepsilon$, such that

$$
\left\|\tilde{u}_{\varepsilon}\right\|_{L^{2}\left(\widetilde{\Omega}_{\varepsilon}\right)^{3}} \leq C a_{\varepsilon}^{2}, \quad\left\|D_{\varepsilon} \tilde{u}_{\varepsilon}\right\|_{L^{2}\left(\widetilde{\Omega}_{\varepsilon}\right)^{3 \times 3}} \leq C a_{\varepsilon}
$$

Proof. Considering $\tilde{u}_{\varepsilon}$ as test function in (4.10) and apply Cauchy-Schwarz's inequality to obtain

$$
\nu\left\|D_{\varepsilon} \tilde{u}_{\varepsilon}\right\|_{L^{2}\left(\widetilde{\Omega}_{\varepsilon}\right)^{3 \times 3}}^{2} \leq\left\|\tilde{f}_{\varepsilon}\right\|_{L^{2}\left(\widetilde{\Omega}_{\varepsilon}\right)^{3}}\left\|\tilde{u}_{\varepsilon}\right\|_{L^{2}\left(\widetilde{\Omega}_{\varepsilon}\right)^{3}} .
$$


Taking into account the assumption on $f_{\varepsilon}$, which in particular does not depend on $x_{3}$, we have

$$
\left\|D_{\varepsilon} \tilde{u}_{\varepsilon}\right\|_{L^{2}\left(\widetilde{\Omega}_{\varepsilon}\right)^{3 \times 3}}^{2} \leq C\left\|\tilde{u}_{\varepsilon}\right\|_{L^{2}\left(\widetilde{\Omega}_{\varepsilon}\right)^{3}},
$$

and from Poincaré's inequality $(5.16)$, we get

$$
\left\|D_{\varepsilon} \tilde{u}_{\varepsilon}\right\|_{L^{2}\left(\widetilde{\Omega}_{\varepsilon}\right)^{3 \times 3}}^{2} \leq C a_{\varepsilon}\left\|D_{\varepsilon} \tilde{u}_{\varepsilon}\right\|_{L^{2}\left(\widetilde{\Omega}_{\varepsilon}\right)^{3 \times 3}}
$$

which proves the second estimate in $(5.19)$. This and again Poincaré's inequality $(5.16)$ give the first one.

The extension of $\left(\tilde{u}_{\varepsilon}, \tilde{p}_{\varepsilon}\right)$ to the whole domain $\Omega$. We extend the velocity $\tilde{u}_{\varepsilon}$ by zero in $\Omega \backslash \widetilde{\Omega}_{\varepsilon}$ (this is compatible with the homogeneous boundary condition on $\partial \Omega_{\varepsilon}$ ), and denote the extension by $\tilde{U}_{\varepsilon}$. Obviously, estimates given in Lemma 5.2 remain valid and the extension $\tilde{U}_{\varepsilon}$ is divergence free too.

In order to extend the pressure $\tilde{p}_{\varepsilon}$ to the whole domain $\Omega$, we recall an important result from [13] which is generalizated to thin domains in [3] and is concerned with the extension of the pressure $p_{\varepsilon}$ to the whole domain $Q_{\varepsilon}$. Thus, we first use a restriction operator $R^{\varepsilon}$ from $H_{0}^{1}\left(Q_{\varepsilon}\right)^{3}$ into $H_{0}^{1}\left(\Omega_{\varepsilon}\right)^{3}$ which is introduced in Lemma 4.5 in [3] as $R_{2}^{\varepsilon}$, next we extend the gradient of the pressure by duality in $H^{-1}\left(Q_{\varepsilon}\right)^{3}$ and finally by means of the dilatation we extend $\tilde{p}_{\varepsilon}$ to $\Omega$.

Lemma 5.3. There exists a (restriction) operator $R^{\varepsilon}$ acting from $H_{0}^{1}\left(Q_{\varepsilon}\right)^{3}$ into $H_{0}^{1}\left(\Omega_{\varepsilon}\right)^{3}$ such that

1. $R^{\varepsilon} \varphi=\varphi$, if $\varphi \in H_{0}^{1}\left(\Omega_{\varepsilon}\right)^{3}$ (elements of $H_{0}^{1}\left(\Omega_{\varepsilon}\right)$ are extended by 0 to $Q_{\varepsilon}$ ).

2. $\operatorname{div} R^{\varepsilon} \varphi=0$ in $\Omega_{\varepsilon}$, if $\operatorname{div} \varphi=0$ on $Q_{\varepsilon}$.

3. For every $\varphi \in H_{0}^{1}\left(Q_{\varepsilon}\right)^{3}$, there exists a positive constant $C$, independent of $\varphi$ and $\varepsilon$, such that

$$
\left\|R^{\varepsilon} \varphi\right\|_{L^{2}\left(\Omega_{\varepsilon}\right)^{3}}+a_{\varepsilon}\left\|D R^{\varepsilon} \varphi\right\|_{L^{2}\left(\Omega_{\varepsilon}\right)^{3 \times 3}} \leq C\left(\|\varphi\|_{L^{2}\left(Q_{\varepsilon}\right)}+a_{\varepsilon}\|D \varphi\|_{L^{2}\left(Q_{\varepsilon}\right)^{3 \times 3}}\right) .
$$

Using the restriction operator $R^{\varepsilon}$ given in Lemma 5.3 , we introduce $F_{\varepsilon}$ in $H^{-1}\left(Q_{\varepsilon}\right)^{3}$ in the following way

$$
\left\langle F_{\varepsilon}, \varphi\right\rangle_{H^{-1}\left(Q_{\varepsilon}\right)^{3}, H_{0}^{1}\left(Q_{\varepsilon}\right)^{3}}=\left\langle\nabla p_{\varepsilon}, R^{\varepsilon} \varphi\right\rangle_{H^{-1}\left(\Omega_{\varepsilon}\right)^{3}, H_{0}^{1}\left(\Omega_{\varepsilon}\right)^{3}}, \quad \text { for any } \varphi \in H_{0}^{1}\left(Q_{\varepsilon}\right)^{3},
$$

and calcule the right hand side of (5.21) by using (4.7), which gives

$$
\left\langle F_{\varepsilon}, \varphi\right\rangle_{H^{-1}\left(Q_{\varepsilon}\right)^{3}, H_{0}^{1}\left(Q_{\varepsilon}\right)^{3}}=-\nu \int_{\Omega_{\varepsilon}} D u_{\varepsilon}: D R^{\varepsilon} \varphi d x+\int_{\Omega_{\varepsilon}} f^{\prime} \cdot\left(R^{\varepsilon} \varphi\right)^{\prime} d x .
$$

Using Lemma 5.2 for fixed $\varepsilon$, we see that it is a bounded functional on $H_{0}^{1}\left(Q_{\varepsilon}\right)$ (se the proof of Lemma 5.4 below), and in fact $F_{\varepsilon} \in H^{-1}\left(Q_{\varepsilon}\right)^{3}$. Moreover, $\operatorname{div} \varphi=0$ implies $\left\langle F_{\varepsilon}, \varphi\right\rangle=0$, and the DeRham theorem gives the existence of $P_{\varepsilon}$ in $L_{0}^{2}\left(Q_{\varepsilon}\right)$ with $F_{\varepsilon}=\nabla P_{\varepsilon}$.

Next, we get for every $\tilde{\varphi} \in H_{0}^{1}(\Omega)^{3}$ where $\tilde{\varphi}\left(x^{\prime}, z_{3}\right)=\varphi\left(x^{\prime}, \varepsilon z_{3}\right)$, using the change of variables $[2.3)$, that

$$
\left\langle\nabla_{\varepsilon} \tilde{P}_{\varepsilon}, \tilde{\varphi}\right\rangle_{H^{-1}(\Omega)^{3}, H_{0}^{1}(\Omega)^{3}}=-\int_{\Omega} \tilde{P}_{\varepsilon} \operatorname{div}_{\varepsilon} \tilde{\varphi} d x^{\prime} d y_{3}=-\varepsilon^{-1} \int_{Q_{\varepsilon}} P_{\varepsilon} \operatorname{div} \varphi d x=\varepsilon^{-1}\left\langle\nabla P_{\varepsilon}, \varphi\right\rangle_{H^{-1}\left(Q_{\varepsilon}\right)^{3}, H_{0}^{1}\left(Q_{\varepsilon}\right)^{3}} .
$$

Using the identification 5.22 of $F_{\varepsilon}$, we have

$$
\left\langle\nabla_{\varepsilon} \tilde{P}_{\varepsilon}, \tilde{\varphi}\right\rangle_{H^{-1}(\Omega)^{3}, H_{0}^{1}(\Omega)^{3}}=\varepsilon^{-1}\left(-\int_{\Omega_{\varepsilon}} D u_{\varepsilon}: D R^{\varepsilon} \varphi d x+\int_{\Omega_{\varepsilon}} f^{\prime}\left(x^{\prime}\right) \cdot\left(R^{\varepsilon} \varphi\right)^{\prime} d x\right),
$$

and applying the change of variables 2.3 , we obtain

$$
\left\langle\nabla_{\varepsilon} \tilde{P}_{\varepsilon}, \tilde{\varphi}\right\rangle_{H^{-1}(\Omega)^{3}, H_{0}^{1}(\Omega)^{3}}=-\int_{\widetilde{\Omega}_{\varepsilon}} D_{\varepsilon} \tilde{u}_{\varepsilon}: D_{\varepsilon} \tilde{R}^{\varepsilon} \tilde{\varphi} d x^{\prime} d y_{3}+\int_{\widetilde{\Omega}_{\varepsilon}} f^{\prime}\left(x^{\prime}\right) \cdot\left(\tilde{R}^{\varepsilon} \tilde{\varphi}\right)^{\prime} d x^{\prime} d y_{3},
$$

where $\tilde{R}^{\varepsilon} \tilde{\varphi}=R^{\varepsilon} \varphi$ for any $\tilde{\varphi} \in H_{0}^{1}(\Omega)^{3}$.

Finally, we estimate the right-hand side of 5.23 and give the estimate of the extended pressure $\tilde{P}_{\varepsilon}$. 
Lemma 5.4. There exists a positive constant $C$ independent of $\varepsilon$, such that

$$
\left\|\tilde{P}_{\varepsilon}\right\|_{L^{2}(\Omega)} \leq C, \quad\left\|\nabla_{\varepsilon} \tilde{P}_{\varepsilon}\right\|_{H^{-1}(\Omega)^{3}} \leq C .
$$

Proof. Applying the dilatation, we have that $\tilde{R}^{\varepsilon} \tilde{\varphi}$ satisfies the following estimates

$$
\begin{aligned}
& \left\|\tilde{R}^{\varepsilon}(\tilde{\varphi})\right\|_{L^{2}\left(\widetilde{\Omega}_{\varepsilon}\right)^{3}} \leq C\left(\|\tilde{\varphi}\|_{L^{2}(\Omega)^{3}}+a_{\varepsilon}\left\|D_{x^{\prime}} \tilde{\varphi}\right\|_{L^{2}(\Omega)^{3 \times 2}}+\frac{a_{\varepsilon}}{\varepsilon}\left\|\partial_{z_{3}} \tilde{\varphi}\right\|_{L^{2}(\Omega)^{3}}\right), \\
& \left\|D_{x^{\prime}} \tilde{R}^{\varepsilon} \tilde{\varphi}\right\|_{L^{2}\left(\widetilde{\Omega}_{\varepsilon}\right)^{3 \times 2}} \leq C\left(\frac{1}{a_{\varepsilon}}\|\tilde{\varphi}\|_{L^{2}(\Omega)^{3}}+\left\|D_{x^{\prime}} \tilde{\varphi}\right\|_{L^{2}(\Omega)^{3 \times 2}}+\frac{1}{\varepsilon}\left\|\partial_{z_{3}} \tilde{\varphi}\right\|_{L^{2}(\Omega)^{3}}\right), \\
& \left\|\partial_{y_{3}} \tilde{R}^{\varepsilon} \tilde{\varphi}\right\|_{L^{2}\left(\widetilde{\Omega}_{\varepsilon}\right)^{3}} \leq C\left(\frac{\varepsilon}{a_{\varepsilon}}\|\tilde{\varphi}\|_{L^{2}(\Omega)^{3}}+\varepsilon\left\|D_{x^{\prime}} \tilde{\varphi}\right\|_{L^{2}(\Omega)^{3 \times 2}}+\left\|\partial_{z_{3}} \tilde{\varphi}\right\|_{L^{2}(\Omega)^{3}}\right) .
\end{aligned}
$$

From the relation 2.1), we have that

$$
\left\|\tilde{R}^{\varepsilon} \tilde{\varphi}\right\|_{L^{2}\left(\widetilde{\Omega}_{\varepsilon}\right)^{3}} \leq C\|\tilde{\varphi}\|_{H_{0}^{1}(\Omega)^{3}}, \quad\left\|D_{\varepsilon} \tilde{R}^{\varepsilon} \tilde{\varphi}\right\|_{L^{2}\left(\widetilde{\Omega}_{\varepsilon}\right)^{3 \times 3}} \leq \frac{C}{a_{\varepsilon}}\|\tilde{\varphi}\|_{H_{0}^{1}(\Omega)^{3}} .
$$

Thus, from Cauchy-Schwarz's inequality and using estimates for $D_{\varepsilon} \tilde{u}_{\varepsilon}$ in (5.19), assumption of $f^{\prime}$ given in (4.11) and estimate of the dilated restricted operator (5.26), we obtain

$$
\begin{aligned}
& \left|\int_{\widetilde{\Omega}_{\varepsilon}} D_{\varepsilon} \tilde{u}_{\varepsilon}: D_{\varepsilon} \tilde{R}^{\varepsilon} \tilde{\varphi} d x^{\prime} d y_{3}\right| \leq C a_{\varepsilon}\left\|D_{\varepsilon} \tilde{R}^{\varepsilon} \tilde{\varphi}\right\|_{L^{2}\left(\widetilde{\Omega}_{\varepsilon}\right)^{3 \times 3}} \leq C\|\tilde{\varphi}\|_{H_{0}^{1}(\Omega)^{3}}, \\
& \left|\int_{\widetilde{\Omega}_{\varepsilon}} f^{\prime} \cdot\left(\tilde{R}^{\varepsilon} \tilde{\varphi}\right)^{\prime} d x^{\prime} d y_{3}\right| \leq C\left\|\tilde{R}^{\varepsilon} \tilde{\varphi}\right\|_{L^{2}\left(\widetilde{\Omega}_{\varepsilon}\right)^{3}} \leq C\|\tilde{\varphi}\|_{H_{0}^{1}(\Omega)^{3}},
\end{aligned}
$$

which together with 5.23 gives

$$
\left|\left\langle\nabla_{\varepsilon} \tilde{P}_{\varepsilon}, \tilde{\varphi}\right\rangle_{H^{-1}(\Omega)^{3}, H_{0}^{1}(\Omega)^{3}}\right| \leq C\|\tilde{\varphi}\|_{H_{0}^{1}(\Omega)^{3}} .
$$

This implies $\left\|\nabla_{\varepsilon} \tilde{P}_{\varepsilon}\right\|_{L^{2}(\Omega)^{3}} \leq C$ and using the Nečas inequality, there exists a representative $\tilde{P}_{\varepsilon} \in L_{0}^{2}(\Omega)$ such that

$$
\left\|\tilde{P}_{\varepsilon}\right\|_{L^{2}(\Omega)} \leq C\left\|\nabla \tilde{P}_{\varepsilon}\right\|_{H^{-1}(\Omega)^{3}} \leq C\left\|\nabla_{\varepsilon} \tilde{P}_{\varepsilon}\right\|_{H^{-1}(\Omega)^{3}},
$$

which implies 5.24.

\subsection{Adaptation of the unfolding method}

The change of variables (2.3) does not provide the information we need about the behavior of of the solution in the microstructure associated to $\widetilde{\Omega}_{\varepsilon}$. To solve this difficulty, we use an adaptation of the unfolding method (for classical versions see [2, 6, 7]) which is related with the change of variables applied in [5] to study the porous part in the case of modelling of a thin film passing a thin porous media. In a simple way, it consists of dividing the domain $\widetilde{\Omega}_{\varepsilon}$ into cubes of lateral length $a_{\varepsilon}$ and vertical length $a_{\varepsilon} / \varepsilon$.

Definition 5.5. Let $\tilde{\varphi}$ be in $L^{2}\left(\widetilde{\Omega}_{\varepsilon}\right)$ and $\tilde{\psi}$ be in $L^{2}(\Omega)$. We define the functions $\hat{\varphi}_{\varepsilon} \in L^{2}\left(\mathbb{R}^{3} \times Y_{f}\right)^{3}$ and $\hat{\psi}_{\varepsilon} \in$ $L^{2}\left(\mathbb{R}^{3} \times Y\right)^{3}$ by

$$
\begin{array}{ll}
\hat{\varphi}_{\varepsilon}\left(x^{\prime}, z_{3}, y\right)=\tilde{\varphi}\left(a_{\varepsilon} \kappa\left(\frac{x^{\prime}}{a_{\varepsilon}}, \frac{\varepsilon z_{3}}{a_{\varepsilon}}\right) e^{\prime}+a_{\varepsilon} y^{\prime}, \frac{a_{\varepsilon}}{\varepsilon} \kappa\left(\frac{x^{\prime}}{a_{\varepsilon}}, \frac{\varepsilon z_{3}}{a_{\varepsilon}}\right) e_{3}+\frac{a_{\varepsilon}}{\varepsilon} y_{3}\right), & \text { a.e. }\left(x^{\prime}, z_{3}, y\right) \in \mathbb{R}^{3} \times Y_{f}, \\
\hat{\psi}_{\varepsilon}\left(x^{\prime}, z_{3}, y\right)=\tilde{\psi}\left(a_{\varepsilon} \kappa\left(\frac{x^{\prime}}{a_{\varepsilon}}, \frac{\varepsilon z_{3}}{a_{\varepsilon}}\right) e^{\prime}+a_{\varepsilon} y^{\prime}, \frac{a_{\varepsilon}}{\varepsilon} \kappa\left(\frac{x^{\prime}}{a_{\varepsilon}}, \frac{\varepsilon z_{3}}{a_{\varepsilon}}\right) e_{3}+\frac{a_{\varepsilon}}{\varepsilon} y_{3}\right), & \text { a.e. }\left(x^{\prime}, z_{3}, y\right) \in \mathbb{R}^{3} \times Y,
\end{array}
$$

assuming $\tilde{\varphi}$ (resp. $\tilde{\psi}$ ) is extended by zero outside $\widetilde{\Omega}_{\varepsilon}$ (resp. $\Omega$ ), where the function $\kappa=\left(\kappa^{\prime}, \kappa_{3}\right)$ is defined by 3.5). 
Remark 5.6. The restrictions of $\hat{\varphi}_{\varepsilon}$ to $\widetilde{Y}_{k, a_{\varepsilon}} \times Y_{f}$ (resp. $\hat{\psi}_{\varepsilon}$ to $\widetilde{Y}_{k, a_{\varepsilon}} \times Y$ ) does not depend on $\left(x^{\prime}, z_{3}\right)$, while as a function of $y$ it is obtained from $(\tilde{\varphi}, \tilde{\psi})$ by using the change of variables

$$
y^{\prime}=\frac{x^{\prime}-a_{\varepsilon} k^{\prime}}{a_{\varepsilon}}, \quad y_{3}=\frac{\varepsilon z_{3}-a_{\varepsilon} k_{3}}{a_{\varepsilon}},
$$

which transforms $\widetilde{Y}_{f_{k}, a_{\varepsilon}}$ into $Y_{f}$ (resp. $\widetilde{Y}_{k, a_{\varepsilon}}$ into $Y$ ).

Proposition 5.7. Let $\tilde{\varphi}$ be in $L^{2}\left(\widetilde{\Omega}_{\varepsilon}\right)$ and $\tilde{\psi}$ be in $L^{2}(\Omega)$. Then, we have

$$
\begin{gathered}
\left\|\hat{\varphi}_{\varepsilon}\right\|_{L^{2}\left(\mathbb{R}^{3} \times Y_{f}\right)^{3}}=\|\tilde{\varphi}\|_{L^{2}\left(\widetilde{\Omega}_{\varepsilon}\right)^{3}}, \quad\left\|D_{y^{\prime}} \hat{\varphi}_{\varepsilon}\right\|_{L^{2}\left(\mathbb{R}^{3} \times Y_{f}\right)^{3 \times 2}}=a_{\varepsilon}\left\|D_{x^{\prime}} \tilde{\varphi}\right\|_{L^{2}\left(\widetilde{\Omega}_{\varepsilon}\right)^{3 \times 2}}, \quad\left\|\partial_{y_{3}} \hat{\varphi}_{\varepsilon}\right\|_{L^{2}\left(\mathbb{R}^{3} \times Y_{f}\right)^{3}}=\frac{a_{\varepsilon}}{\varepsilon}\left\|\partial_{z_{3}} \tilde{\varphi}\right\|_{L^{2}\left(\widetilde{\Omega}_{\varepsilon}\right)^{3}}, \\
\left\|\hat{\psi}_{\varepsilon}\right\|_{L^{2}\left(\mathbb{R}^{3} \times Y\right)^{3}}=\|\tilde{\psi}\|_{L^{2}(\Omega)^{3}}, \quad\left\|D_{y^{\prime}} \hat{\psi}_{\varepsilon}\right\|_{L^{2}\left(\mathbb{R}^{3} \times Y\right)^{3 \times 2}}=a_{\varepsilon}\left\|D_{x^{\prime}} \tilde{\psi}\right\|_{L^{2}(\Omega)^{3 \times 2}}, \quad\left\|\partial_{y_{3}} \hat{\psi}_{\varepsilon}\right\|_{L^{2}\left(\mathbb{R}^{3} \times Y\right)^{3}}=\frac{a_{\varepsilon}}{\varepsilon}\left\|\partial_{z_{3}} \tilde{\psi}\right\|_{L^{2}(\Omega)^{3}} .
\end{gathered}
$$

Proof. We will only make the proof for $\hat{\varphi}_{\varepsilon}$. The procedure for $\hat{\psi}$ is similar, so we omit it. Taking into account the definition (5.27) of $\hat{\varphi}_{\varepsilon}$, we obtain

$$
\begin{aligned}
\int_{\mathbb{R}^{3} \times Y_{f}}\left|D_{y^{\prime}} \hat{\varphi}_{\varepsilon}\left(x^{\prime}, z_{3}, y\right)\right|^{2} d x^{\prime} d z_{3} d y & =\sum_{k \in \mathbb{R}^{3}} \int_{\widetilde{Y}_{k, a_{\varepsilon}}} \int_{Y_{f}}\left|D_{y^{\prime}} \hat{\varphi}_{\varepsilon}\left(x^{\prime}, z_{3}, y\right)\right|^{2} d x^{\prime} d z_{3} d y \\
& =\sum_{k \in \mathbb{R}^{3}} \int_{\widetilde{Y}_{k, a_{\varepsilon}}} \int_{Y_{f}}\left|D_{y^{\prime}} \tilde{\varphi}\left(a_{\varepsilon} k^{\prime}+a_{\varepsilon} y^{\prime}, a_{\varepsilon} \varepsilon^{-1} k_{3}+a_{\varepsilon} \varepsilon^{-1} y_{3}\right)\right|^{2} d x^{\prime} d z_{3} d y .
\end{aligned}
$$

We observe that $\tilde{u}_{\varepsilon}$ does not depend on $\left(x^{\prime}, z_{3}\right)$, then we can deduce

$$
\int_{\mathbb{R}^{3} \times Y_{f}}\left|D_{y^{\prime}} \hat{\varphi}_{\varepsilon}\left(x^{\prime}, z_{3}, y\right)\right|^{2} d x^{\prime} d z_{3} d y=\frac{a_{\varepsilon}^{3}}{\varepsilon} \sum_{k \in \mathbb{R}^{3}} \int_{Y_{f}}\left|D_{y^{\prime}} \tilde{\varphi}\left(a_{\varepsilon} k^{\prime}+a_{\varepsilon} y^{\prime}, a_{\varepsilon} \varepsilon^{-1} k_{3}+a_{\varepsilon} \varepsilon^{-1} y_{3}\right)\right|^{2} d y .
$$

By the change of variables 5.29 , we obtain

$$
\begin{aligned}
\int_{\mathbb{R}^{3} \times Y_{f}}\left|D_{y^{\prime}} \hat{\varphi}_{\varepsilon}\left(x^{\prime}, z_{3}, y\right)\right|^{2} d x^{\prime} d z_{3} d y & =a_{\varepsilon}^{2} \sum_{k \in \mathbb{R}^{3}} \int_{\widetilde{Y}_{f_{k}, a_{\varepsilon}}}\left|D_{x^{\prime}} \tilde{\varphi}\left(x^{\prime}, z_{3}\right)\right|^{2} d x^{\prime} d z_{3} \\
& =a_{\varepsilon}^{2} \int_{\widetilde{\Omega}_{\varepsilon}}\left|D_{x^{\prime}} \tilde{\varphi}\left(x^{\prime}, z_{3}\right)\right|^{2} d x^{\prime} d z_{3} .
\end{aligned}
$$

Thus, we get the property for $D_{y^{\prime}} \hat{\varphi}_{\varepsilon}$.

Similarly, we have

$$
\int_{\mathbb{R}^{3} \times Y_{f}}\left|\partial_{y_{3}} \hat{\varphi}_{\varepsilon}\left(x^{\prime}, z_{3}, y\right)\right|^{2} d x^{\prime} d z_{3} d y=\frac{a_{\varepsilon}^{3}}{\varepsilon} \sum_{k \in \mathbb{R}^{3}} \int_{Y_{f}}\left|\partial_{y_{3}} \tilde{\varphi}\left(a_{\varepsilon} k^{\prime}+a_{\varepsilon} y^{\prime}, a_{\varepsilon} \varepsilon^{-1} k_{3}+a_{\varepsilon} \varepsilon^{-1} y_{3}\right)\right|^{2} d y .
$$

By the change of variables 5.29 we obtain

$$
\begin{aligned}
\int_{\mathbb{R}^{3} \times Y_{f}}\left|\partial_{y_{3}} \hat{\varphi}_{\varepsilon}\left(x^{\prime}, z_{3}, y\right)\right|^{2} d x^{\prime} d z_{3} d y & =\frac{a_{\varepsilon}^{2}}{\varepsilon^{2}} \sum_{k \in \mathbb{R}^{3}} \int_{\widetilde{Y}_{f_{k}, a_{\varepsilon}}}\left|\partial_{y_{3}} \tilde{\varphi}\left(x^{\prime}, z_{3}\right)\right|^{2} d x^{\prime} d z_{3} . \\
& =\frac{a_{\varepsilon}^{2}}{\varepsilon^{2}} \int_{\widetilde{\Omega}_{\varepsilon}}\left|\partial_{y_{3}} \tilde{\varphi}\left(x^{\prime}, z_{3}\right)\right|^{2} d x^{\prime} d z_{3},
\end{aligned}
$$

so the the property for $\partial_{y_{3}} \hat{\varphi}_{\varepsilon}$ is proved. Finally, reasoning analogously we deduce

$$
\int_{\mathbb{R}^{3} \times Y_{f}}\left|\hat{\varphi}_{\varepsilon}\left(x^{\prime}, z_{3}, y\right)\right|^{2} d x^{\prime} d z_{3} d y=\int_{\widetilde{\Omega}_{\varepsilon}}\left|\tilde{\varphi}\left(x^{\prime}, z_{3}\right)\right|^{2} d x^{\prime} d z_{3},
$$

and the property for $\hat{\varphi}_{\varepsilon}$ holds. 
Now, from functions $\tilde{u}_{\varepsilon}$ and $\tilde{P}_{\varepsilon}$ we define $\hat{u}_{\varepsilon}$ by using (5.27) and $\hat{P}_{\varepsilon}$ by means of (5.28). Below, we get the estimates for these sequences.

Lemma 5.8. There exists a constant $C>0$ independent of $\varepsilon$, such that $\hat{u}_{\varepsilon}$ defined by (5.27) and $\hat{P}_{\varepsilon}$ defined by (5.28) satisfy

$$
\begin{gathered}
\left\|\hat{u}_{\varepsilon}\right\|_{L^{2}\left(\mathbb{R}^{3} \times Y_{f}\right)^{3}} \leq C a_{\varepsilon}^{2}, \quad\left\|D_{y} \hat{u}_{\varepsilon}\right\|_{L^{2}\left(\mathbb{R}^{3} \times Y_{f}\right)^{3 \times 3}} \leq C a_{\varepsilon}^{2}, \\
\left\|\hat{P}_{\varepsilon}\right\|_{L^{2}\left(\mathbb{R}^{3} \times Y\right)} \leq C .
\end{gathered}
$$

Proof. Estimates (5.30 and 5.31) easily follow from Proposition 5.7 and estimates given in Lemmas 5.2 and 5.4

To finish this section, we will give the variational formulation satisfied by the functions $\left(\hat{u}_{\varepsilon}, \hat{P}_{\varepsilon}\right)$, which will be useful in the following sections. Thus, we consider $\varphi_{\varepsilon}\left(x^{\prime}, z_{3}\right)=\varphi\left(x^{\prime}, z_{3}, x^{\prime} / a_{\varepsilon}, \varepsilon z_{3} / a_{\varepsilon}\right)$ as test function in (4.10) where $\varphi\left(x^{\prime}, z_{3}, y\right) \in C_{c}^{1}\left(\Omega ; H_{\mathrm{per}}^{1}(Y)^{3}\right)$. Taking into account the extension of the pressure

$$
\int_{\widetilde{\Omega}_{\varepsilon}} \nabla_{\varepsilon} \tilde{p}_{\varepsilon} \cdot \varphi_{\varepsilon} d x^{\prime} d z_{3}=\int_{\Omega} \nabla_{\varepsilon} \tilde{P}_{\varepsilon} \cdot \varphi_{\varepsilon}, d x^{\prime} d z_{3},
$$

the variational formulation 4.10 reads

$$
\int_{\widetilde{\Omega}_{\varepsilon}} D_{\varepsilon} \tilde{u}_{\varepsilon}: D_{\varepsilon} \varphi_{\varepsilon} d x^{\prime} d y_{3}-\int_{\Omega} \tilde{P}_{\varepsilon} \operatorname{div}_{\varepsilon} \varphi_{\varepsilon} d x^{\prime} d y_{3}=\int_{\widetilde{\Omega}_{\varepsilon}} f^{\prime} \cdot \varphi_{\varepsilon}^{\prime} d x^{\prime} d y_{3}
$$

By the change of variables given in Remark 5.6, we obtain

$$
\begin{aligned}
& \frac{\nu}{a_{\varepsilon}^{2}} \int_{\Omega \times Y_{f}} D_{y} \hat{u}_{\varepsilon}: D_{y} \varphi d x^{\prime} d z_{3} d y-\int_{\Omega \times Y} \hat{P}_{\varepsilon} \operatorname{div}_{\varepsilon} \varphi d x^{\prime} d z_{3} d y-\frac{1}{a_{\varepsilon}} \int_{\Omega \times Y} \hat{P}_{\varepsilon} \operatorname{div}_{y} \varphi d x^{\prime} d z_{3} d y \\
& =\int_{\Omega \times Y_{f}} f^{\prime} \cdot \varphi^{\prime} d x^{\prime} d z_{3} d y+O_{\varepsilon},
\end{aligned}
$$

When $\varepsilon$ tends to zero, we will analyze the asymptotic behavior of sequence $\left(\hat{u}_{\varepsilon}, \hat{P}_{\varepsilon}\right)$ in the next sections.

\subsection{Some compactness results}

In this subsection we obtain some compactness results concerning the behavior of the sequences $\left(\tilde{U}_{\varepsilon}, \tilde{P}_{\varepsilon}\right)$ and $\left(\hat{u}_{\varepsilon}, \hat{p}_{\varepsilon}\right)$.

Lemma 5.9. For a subsequence of $\varepsilon$ still denoted by $\varepsilon$, there exist $u \in L^{2}(\Omega)^{3}$ and $\hat{u} \in L^{2}\left(\mathbb{R}^{3} ; H_{\mathrm{per}}^{1}\left(Y_{f}\right)^{3}\right)$, such that

$$
\begin{gathered}
a_{\varepsilon}^{-2} \tilde{U}_{\varepsilon} \rightarrow\left(u^{\prime}, 0\right) \text { in } L^{2}(\Omega)^{3}, \\
a_{\varepsilon}^{-2} \hat{u}_{\varepsilon} \rightarrow \hat{u} \text { in } L^{2}\left(\mathbb{R}^{3} ; H^{1}\left(Y_{f}\right)^{3}\right), \quad a_{\varepsilon}^{-2} D_{y} \hat{u}_{\varepsilon} \rightarrow D_{y} \hat{u} \text { in } L^{2}\left(\mathbb{R}^{3} \times Y_{f}\right)^{3},
\end{gathered}
$$

with the boundary conditions $u=0$ on $z_{3}=\{0,1\}$ and $\hat{u}=0$ in $\Omega \times T$ and in $\left(\mathbb{R}^{3} \backslash \Omega\right) \times Y_{f}$.

Moreover, defining $U\left(x^{\prime}\right)=\int_{0}^{1} u\left(x^{\prime}, z_{3}\right) d z_{3}$ and $\hat{U}\left(x^{\prime}, y\right)=\int_{0}^{1} \hat{U}\left(x^{\prime}, z_{3}, y\right) d z_{3}$, it holds

$$
U\left(x^{\prime}\right)=\int_{Y_{f}} \hat{U}\left(x^{\prime}, y\right) d y \quad \text { with } \quad \int_{Y_{f}} \hat{U}_{3}\left(x^{\prime}, y\right) d y=0 \quad \text { in } \omega,
$$

and $\hat{U}\left(x^{\prime}, y\right) \in L^{2}\left(\mathbb{R}^{2} ; H_{\mathrm{per}}^{1}\left(Y_{f}\right)^{3}\right)$ satisfies $\hat{U}=0$ in $\mathbb{R}^{2} \times T$ and in $\left(\mathbb{R}^{2} \backslash \omega\right) \times Y_{f}$ together with divergence conditions

$$
\begin{gathered}
\operatorname{div}_{y} \hat{U}\left(x^{\prime}, y\right)=0 \quad \text { in } \mathbb{R}^{3} \times Y_{f}, \\
\operatorname{div}_{x^{\prime}}\left(\int_{Y_{f}} \hat{U}^{\prime}\left(x^{\prime}, y\right) d y\right)=0 \quad \text { in } \mathbb{R}^{2}, \\
\left(\int_{Y_{f}} \hat{U}^{\prime}\left(x^{\prime}, y\right) d y\right) \cdot n=0 \quad \text { on } \partial \omega,
\end{gathered}
$$


Proof. We start with the extended velocity $\tilde{U}_{\varepsilon}$. From estimates 5.19 , we get

$$
\left\|\tilde{U}_{\varepsilon}\right\|_{L^{2}(\Omega)^{3}} \leq C a_{\varepsilon}^{2}, \quad\left\|D_{x^{\prime}} \tilde{U}_{\varepsilon}\right\|_{L^{2}\left(Q_{\varepsilon}\right)^{3}} \leq C a_{\varepsilon}, \quad\left\|\partial_{y_{3}} \tilde{U}_{\varepsilon}\right\|_{L^{2}\left(Q_{\varepsilon}\right)^{3}} \leq C a_{\varepsilon} \varepsilon .
$$

Then, applying Lema 3.12-(ii) in [4, we get the existence of $u \in L^{2}(\Omega)^{3}$ with $u_{3}=0$ on $z_{3}=\{0,1\}$, such that it holds convergence (5.34), up to a subsequence, and the following divergence condition

$$
\operatorname{div}_{x^{\prime}} U^{\prime}\left(x^{\prime}\right)=0 \quad \text { in } \omega, \quad U^{\prime}\left(x^{\prime}\right) \cdot n=0 \quad \text { in } \partial \omega .
$$

Next, taking into account that from estimates of the velocity $\hat{u}_{\varepsilon}$ given in $(5.30)$ we have the existence of $\hat{u} \in L^{2}\left(\mathbb{R}^{3} ; H_{\text {per }}^{1}\left(Y_{f}\right)^{3}\right)$ satisfying, up to a subsequence, convergences 5.35. Taking into account that $a_{\varepsilon}^{-2} \hat{u}_{\varepsilon}$ vanishes on $\widetilde{\Omega}_{\varepsilon} \times T$, we deduce that $\hat{u}$ also vanishes on $\Omega \times T$. Moreover, by construction $\hat{u}_{\varepsilon}$ is zero outside $\widetilde{\Omega}_{\varepsilon}$ and so $\hat{u}$ vanishes on $\left(\mathbb{R}^{3} \backslash \Omega\right) \times Y_{f}$. Since $\operatorname{div}_{\varepsilon} \tilde{u}_{\varepsilon}=0$ in $\widetilde{\Omega}_{\varepsilon}$, by applying the change of variables 5.29 , we get

$$
a_{\varepsilon}^{-1} \operatorname{div}_{y} \hat{u}_{\varepsilon}=0 .
$$

Multiplying by $a_{\varepsilon}^{-1}$ and passing to the limit by using convergence 5.35 , we deduce $\operatorname{div}_{y} \hat{u}=0$ in $\mathbb{R}^{3} \times Y_{f}$ and so we get (5.37).

It remains to prove that $\hat{u}$ is periodic in $y$. Thus follows by passing to the limit in the equality

$$
a_{\varepsilon}^{-2} \hat{u}_{\varepsilon}\left(x^{\prime}+a_{\varepsilon} e_{1}, z_{3},-\frac{1}{2}, y_{2}, y_{3}\right)=a_{\varepsilon}^{-2} \hat{u}_{\varepsilon}\left(x^{\prime}, z_{3}, \frac{1}{2}, y_{2}, y_{3}\right)
$$

which is a consequence of definition (5.27). This shows

$$
\hat{u}\left(x^{\prime}, z_{3},-\frac{1}{2}, y_{2}, y_{3}\right)=\hat{u}\left(x^{\prime}, z_{3}, \frac{1}{2}, y_{2}, y_{3}\right),
$$

and then is proved the periodicity of $\hat{u}$ with respect to $y_{1}$. Similarly, we prove the periodicity with respect to $y_{2}$. To prove the periodicity with respect to $y_{3}$, we consider

$$
a_{\varepsilon}^{-2} \hat{u}_{\varepsilon}\left(x^{\prime}, z_{3}+\frac{a_{\varepsilon}}{\varepsilon}, y_{1}, y_{2},-\frac{1}{2}\right)=a_{\varepsilon}^{-2} \hat{u}_{\varepsilon}\left(x^{\prime}, z_{3}, y_{1}, y_{2}, \frac{1}{2}\right),
$$

and passing to the limit we have

$$
\hat{u}_{\varepsilon}\left(x^{\prime}, z_{3}, y_{1}, y_{2},-\frac{1}{2}\right)=a_{\varepsilon}^{-2} \hat{u}_{\varepsilon}\left(x^{\prime}, z_{3}, y_{1}, y_{2}, \frac{1}{2}\right),
$$

which shows the periodicity with respect to $y_{3}$.

To finish, we prove relation 5.36. To do this, from the change of variables 5.29, we have

$$
\int_{\widetilde{\Omega}_{\varepsilon}} \tilde{u}_{\varepsilon}\left(x^{\prime}, z_{3}\right) d x^{\prime} d z_{3}=\int_{\Omega \times Y_{f}} \hat{u}_{\varepsilon}\left(x^{\prime}, z_{3}, y\right) d x^{\prime} d z_{3} d y .
$$

By using extension of the velocity and the dilatation (2.3), we get

$$
\int_{\Omega} \tilde{U}_{\varepsilon}\left(x^{\prime}, z_{3}\right) d x^{\prime} d z_{3}=\int_{\Omega \times Y_{f}} \hat{u}_{\varepsilon}\left(x^{\prime}, z_{3}, y\right) d x^{\prime} d z_{3} d y .
$$

Multiplying this equality by $a_{\varepsilon}^{-2}$, passing to the limit by using convergences 5.35 and 5.34, we deduce

$$
\int_{\Omega} u\left(x^{\prime}, z_{3}\right) d z_{3}=\int_{\Omega \times Y_{f}} \hat{u}\left(x^{\prime}, z_{3}, y\right) d y .
$$

which, taking into account that $u_{3}=0$ in $\Omega$, implies relation (5.36). Finally, this together with relation (5.40) gives the divergence condition (5.38). Finally, condition $\hat{u}=0$ in $\left(\mathbb{R}^{3} \backslash \Omega\right) \times Y_{f}$ implies $\hat{U}=0$ in $\left(\mathbb{R}^{2} \backslash \omega\right) \times Y_{f}$. This and 5.38 imply 5.39 . 
Lemma 5.10. For a subsequence of $\varepsilon$ still denoted by $\varepsilon$, there exists $p \in L_{0}^{2}(\omega)$ independent of $y_{3}$, such that

$$
\begin{gathered}
\tilde{P}_{\varepsilon} \rightarrow p \quad \text { in } L^{2}(\Omega), \\
\hat{p}_{\varepsilon} \rightarrow p \quad \text { in } L^{2}\left(\mathbb{R}^{2} \times Y\right)^{2} .
\end{gathered}
$$

Proof. Taking into account estimate the first estimate in 5.24 and (5.31), we deduce that there exist $p \in L^{2}(\Omega)$ and $\hat{p} \in L^{2}(\Omega \times Y)$ such that, up to a subsequence,

$$
\begin{gathered}
\tilde{P}_{\varepsilon} \rightarrow p \quad \text { in } L^{2}(\Omega), \\
\hat{p}_{\varepsilon} \rightarrow p \quad \text { in } L^{2}\left(\mathbb{R}^{2} \times Y\right) .
\end{gathered}
$$

From convergence 5.43 we deduce that $\partial_{y_{3}} \tilde{P}_{\varepsilon}$ also converges to $\partial_{y_{3}} p$ in $H^{-1}(\Omega)$. Also, from the second estimate in 5.24 , we can deduce that by noting that $\partial_{z_{3}} \tilde{P}_{\varepsilon}$ converges to zero in in $H^{-1}(\Omega)$. Then, by the uniqueness of the limit we obtain $\partial_{z_{3}} p=0$ and so $\tilde{p}$ is independent of $z_{3}$. Since $\tilde{p}_{\varepsilon}$ has null mean value in $\widetilde{\Omega}_{\varepsilon}$, then $p$ has null mean value in $\omega$. Moreover, reasoning similarly to obtain relation (5.36), it follows the relation $\tilde{p}\left(x^{\prime}\right)=\int_{Y} \hat{p}\left(x^{\prime}, z_{3}, y\right) d y$.

Finally, we shall prove that $\hat{p}$ is in fact equal to $p$ in $\omega$. To do that, it remains to prove that $\hat{p}$ does not depend on $y$. To do this we multiply by $a_{\varepsilon}$ the variational formulation (5.33), which gives

$$
\begin{aligned}
& \frac{\nu}{a_{\varepsilon}} \int_{\Omega \times Y_{f}} D_{y} \hat{u}_{\varepsilon}: D_{y} \varphi d x^{\prime} d z_{3} d y-a_{\varepsilon} \int_{\Omega \times Y} \hat{P}_{\varepsilon} \operatorname{div}_{x^{\prime}} \varphi^{\prime} d x^{\prime} d z_{3} d y-\frac{a_{\varepsilon}}{\varepsilon} \int_{\Omega \times Y} \hat{P}_{\varepsilon} \partial_{z_{3}} \varphi_{3} d x^{\prime} d z_{3} d y \\
& -\int_{\Omega \times Y} \hat{P}_{\varepsilon} \operatorname{div}_{y} \varphi d x^{\prime} d z_{3} d y=a_{\varepsilon} \int_{\Omega \times Y_{f}} f^{\prime} \cdot \varphi^{\prime} d x^{\prime} d z_{3} d y+O_{\varepsilon},
\end{aligned}
$$

Thus, by using relation (2.1) and convergences (5.35) and (5.41) and (5.41), we pass to the limit when $\varepsilon$ tends to zero and we get

$$
\int_{\Omega \times Y} \hat{p} \operatorname{div}_{y} \varphi d x^{\prime} d z_{3} d y=0, \quad \forall \varphi \in C_{c}^{1}\left(\Omega ; H_{\mathrm{per}}^{1}(Y)^{3}\right),
$$

By density it holds in $L^{2}\left(\Omega ; H_{\text {per }}^{1}(Y)^{3}\right)$ and implies that $p$ does not depend on the variable $y$.

Finally, following [13] adapted to the case of thin domains, we prove that the convergence of the pressure is in fact strong. As $\tilde{u}_{3}=0$ and $\tilde{p}$ only depends on $x^{\prime}$, let $\sigma_{\varepsilon}\left(x^{\prime}, z_{3}\right)=\left(\sigma_{\varepsilon}^{\prime}\left(x^{\prime}\right), 0\right) \in H_{0}^{1}(\omega)^{3}$ be such that

$$
\sigma_{\varepsilon} \rightarrow \sigma \quad \text { in } H_{0}^{1}(\omega)^{3} \text {. }
$$

Then, we have

$$
\begin{aligned}
\left|<\nabla_{\varepsilon} \tilde{P}_{\varepsilon}, \sigma_{\varepsilon}>_{H^{-1}(\Omega), H_{0}^{1}(\Omega)}-<\nabla_{x^{\prime}} p, \sigma>_{H^{-1}(\Omega), H_{0}^{1}(\Omega)}\right| \leq & \left|<\nabla_{\varepsilon} \tilde{P}_{\varepsilon}, \sigma_{\varepsilon}-\sigma>_{H^{-1}(\Omega), H_{0}^{1}(\Omega)}\right| \\
& +\left|<\nabla_{\varepsilon} \tilde{P}_{\varepsilon}-\nabla_{x^{\prime}} p, \sigma>_{H^{-1}(\Omega), H_{0}^{1}(\Omega)}\right| .
\end{aligned}
$$

On the one hand, using convergence $\sqrt{5.10}$, we have

$$
\left|<\nabla_{\varepsilon} \tilde{P}_{\varepsilon}-\nabla_{x^{\prime}} p, \sigma>_{\Omega}\right|=\left|\int_{\Omega}\left(\tilde{P}_{\varepsilon}-p\right) \operatorname{div}_{x^{\prime}} \sigma^{\prime} d x\right| \rightarrow 0, \quad \text { as } \varepsilon \rightarrow 0 .
$$

On the other hand, from 5.23) and proceeding as in the proof of Lemma 5.4, we have

$$
\begin{aligned}
\left|<\nabla_{\varepsilon} \tilde{P}_{\varepsilon}, \sigma_{\varepsilon}-\sigma>_{\Omega}\right|= & \left|<\nabla_{x^{\prime}} \tilde{P}_{\varepsilon}, \tilde{R}^{\varepsilon}\left(\sigma_{\varepsilon}^{\prime}-\sigma^{\prime}\right)>_{\tilde{\Omega}_{\varepsilon}}\right| \\
& \leq C\left(\left\|\sigma_{\varepsilon}^{\prime}-\sigma^{\prime}\right\|_{L^{2}(\omega)^{3}}+a_{\varepsilon}\left\|D_{x^{\prime}}\left(\sigma_{\varepsilon}^{\prime}-\sigma^{\prime}\right)\right\|_{L^{2}(\omega)^{3 \times 2}}\right) \rightarrow 0 \quad \text { as } \varepsilon \rightarrow 0,
\end{aligned}
$$

by virtue of $\left(5.45\right.$ and the Rellich theorem. This implies that $\nabla_{\varepsilon} \tilde{P}_{\varepsilon} \rightarrow \nabla_{x^{\prime}} p$ strongly in $H^{-1}(\Omega)^{3}$, which together the Nečas inequality, implies the strong convergence of the pressure $\tilde{P}_{\varepsilon}$ given in 5.41 . This and and the first relation given in 5.7 prove that $\hat{P}_{\varepsilon}$ also converges strongly to $p$. 


\subsection{Proof of main result}

In this subsection, we describe the asymptotic behavior of solution of problem (4.6) by using the convergences given in Lemmas 5.9 and 5.10 . First, we give the homogenized system satisfied by the unfolding functions and then, we introduce the local problems and give the homogenized flow model.

Proof of Theorem 4.1. We divide the proof in two steps.

Step 1. From Lemmas 5.9 and 5.10 we have that there exist $\hat{u} \in L^{2}\left(\mathbb{R}^{2} ; H_{\text {per }}^{1}\left(Y_{f}\right)^{3}\right)$ and $p \in L_{0}^{2}(\omega)$ as the limits of $a_{\varepsilon}^{-2} \hat{u}_{\varepsilon}$ and $\hat{P}_{\varepsilon}$ satisfying convergences 5.35 and 5.42 respectively. In this step, we prove that $(\hat{U}, p)$ satisfies the following homogenized system

$$
\left\{\begin{aligned}
-\nu \Delta_{z} \hat{U}+\nabla_{z} \hat{q}=f^{\prime}-\nabla_{x^{\prime}} p & \text { in } \omega \times Y_{f} \\
\operatorname{div}_{y} \hat{U}=0 & \text { in } \omega \times Y_{f} \\
\operatorname{div}_{x^{\prime}}\left(\int_{Y_{f}} \hat{U}^{\prime} d y\right)=0 & \text { in } \omega \\
\left(\int_{Y_{f}} \hat{U}^{\prime} d y\right) \cdot n=0 & \text { on } \partial \omega \\
\hat{U}=0 & \text { in } \omega \times T \\
\hat{q} \in L^{2}\left(\omega ; L_{\mathrm{per}}^{2}(Y) / \mathbb{R}\right) . &
\end{aligned}\right.
$$

Taking into account Lemma 5.9 , we have that $\hat{U}$ satisfies conditions $5.46,2,3,4,5$.

Now, we want to prove that $(\hat{U}, p)$ satisfies equation $5.46 n_{1}$. To do this, we consider $\varphi_{\varepsilon}\left(x^{\prime}, z_{3}\right)=\left(\varphi_{\varepsilon}^{\prime}, \varphi_{\varepsilon, 3}\right)$ with $\varphi_{\varepsilon}^{\prime}=\varphi^{\prime}\left(x^{\prime}, z_{3}, x^{\prime} / a_{\varepsilon}, \varepsilon z_{3} / a_{\varepsilon}\right)$ and $\varphi_{\varepsilon, 3}=\varepsilon \varphi_{3}\left(x^{\prime}, z_{3}, x^{\prime} / a_{\varepsilon}, \varepsilon z_{3} / a_{\varepsilon}\right)$ as test function in (5.33) where $\varphi\left(x^{\prime}, z_{3}, y\right) \in$ $C_{c}^{1}\left(\Omega ; H_{\mathrm{per}}^{1}(Y)^{3}\right)$ such that $\varphi=0$ in $\Omega \times T, \operatorname{div}_{x^{\prime}}\left(\int_{Y_{f}} \varphi^{\prime} d y\right)=0$ in $\Omega$ and $\operatorname{div}_{y} \varphi=0$ in $\Omega \times Y_{f}$. Then, the variational formulation reads

$$
\begin{aligned}
& \frac{\nu}{a_{\varepsilon}^{2}} \int_{\Omega \times Y_{f}} D_{y} \hat{u}_{\varepsilon}: D_{y} \varphi d x^{\prime} d z_{3} d y-\int_{\Omega \times Y} \hat{P}_{\varepsilon} \operatorname{div}_{x^{\prime}} \varphi^{\prime} d x^{\prime} d z_{3} d y-\int_{\Omega \times Y} \hat{P}_{\varepsilon} \partial_{z_{3}} \varphi_{3} d x^{\prime} d z_{3} d y \\
& =\int_{\Omega \times Y_{f}} f^{\prime} \cdot \varphi^{\prime} d x^{\prime} d z_{3} d y+O_{\varepsilon},
\end{aligned}
$$

We pass to the limit by using convergences 5.35 and 5.42 and we obtain

$$
\nu \int_{\Omega \times Y_{f}} D_{y} \hat{u}: D_{y} \varphi d x^{\prime} d z_{3} d y-\int_{\Omega \times Y} p \operatorname{div}_{x^{\prime}} \varphi^{\prime} d x^{\prime} d z_{3} d y-\int_{\Omega \times Y} p \partial_{z_{3}} \varphi_{3} d x^{\prime} d z_{3} d y=\int_{\Omega \times Y_{f}} f^{\prime} \cdot \varphi^{\prime} d x^{\prime} d z_{3} d y
$$

Taking into account that $p$ does not depend on $z_{3}$, we have that

$$
\int_{\Omega \times Y} p \operatorname{div}_{x^{\prime}} \varphi^{\prime} d x^{\prime} d z_{3} d y=\int_{\Omega} p\left(\operatorname{div}_{x^{\prime}} \int_{Y_{f}} \varphi^{\prime} d y\right) d x^{\prime} d z_{3}=0 \quad \text { and } \quad \int_{\Omega \times Y} p \partial_{z_{3}} \varphi_{3} d x^{\prime} d z_{3} d y=0
$$

Then we obtain

$$
\nu \int_{\Omega \times Y_{f}} D_{y} \hat{u}: D_{y} \varphi d x^{\prime} d z_{3} d y=\int_{\Omega \times Y_{f}} f^{\prime} \cdot \varphi^{\prime} d x^{\prime} d z_{3} d y
$$

We take into account that there is no $z_{3}$-dependence in the obtained variational formulation. For that, we can consider $\varphi$ independent of $z_{3}$, which implies that $(\hat{U}, p)$ satisfies the same variational formulation with integrals in 
$\omega \times Y_{f}$. By density, this equality holds for every function in the Hilbert space $V$ defined by

$$
V=\left\{\begin{array}{l}
\varphi\left(x^{\prime}, y\right) \in L^{2}\left(\omega ; H_{\mathrm{per}}^{1}(Y)^{3}\right) \text { such that } \\
\operatorname{div}_{x^{\prime}}\left(\int_{Y_{f}} \varphi\left(x^{\prime}, y\right) d y\right)=0 \text { in } \omega, \quad\left(\int_{Y_{f}} \varphi\left(x^{\prime}, y\right) d y\right) \cdot n=0 \text { on } \partial \omega \\
\operatorname{div}_{y} \varphi\left(x^{\prime}, y\right)=0 \text { in } \omega \times Y_{f}, \quad \varphi\left(x^{\prime}, y\right)=0 \text { in } \omega \times T
\end{array}\right\} .
$$

By Lax-Milgram lemma, the variational formulation (5.47) in the Hilbert $V$ admits a unique solution $\hat{u}$ in $V$. Reasoning as in 11, the orthogonal of $V$ with respect to the usual scalar product in $L^{2}(\omega \times Y)$ is made of gradients of the form $\nabla_{x^{\prime}} q\left(x^{\prime}\right)+\nabla_{z} \hat{q}\left(x^{\prime}, z\right)$, with $q\left(x^{\prime}\right) \in L^{2}(\omega) / \mathbb{R}$ and $\hat{q}\left(x^{\prime}, y\right) \in L^{2}\left(\omega ; L_{\text {per }}^{2}(Y) / \mathbb{R}\right)$. Therefore, since $\hat{U} \in$ $L^{2}\left(\omega ; H_{\text {per }}^{1}\left(Y_{f}\right)^{3}\right), \hat{U}=0$ in $\omega \times T, \operatorname{div}_{y} \hat{U}=0$ in $\omega \times Y_{f}$, by integration by parts we deduce that $\hat{U}, p$ and $\hat{q}$ satisfy system (5.46). It remains to prove that $q$ coincides with pressure $p$. This can be easily done by multiplying the variational formulation (5.33) by a test function $\varphi$ independent of $z_{3}$ with $\operatorname{div}_{y}$ equal to zero, and identifying limits.

Step 2. In this step we deduce the expression for velocity $U$ given in 4.12 and the Darcy equation satisfied by $p$ given in 4.13). To do this, let us define the local problems which are useful to eliminate the variable $y$ of the previous homogenized problem and then obtain a Darcy equation for the pressure $p$.

Thus, we consider $\left(w^{i}, \pi^{i}\right), i=1,2,3$, as the unique solution of problem [4.14), see [13, where we observe that

$$
w^{3}=0 \quad \text { in } H_{\mathrm{per}}^{1}\left(Y_{f}\right)^{3}, \quad \pi^{3}=y_{3} \quad \text { in } L_{\mathrm{per}}^{2}\left(Y_{f}\right) / \mathbb{R} .
$$

Then, reasoning by linearity and uniqueness, we deduce

$$
\hat{U}\left(x^{\prime}, y\right)=\sum_{i=1}^{2}\left(f_{i}^{\prime}\left(x^{\prime}\right)-\partial_{x_{i}} p\left(x^{\prime}\right)\right) w^{i}(y), \quad \hat{q}\left(x^{\prime}, y\right)=\sum_{i=1}^{2}\left(f_{i}^{\prime}\left(x^{\prime}\right)-\partial_{x_{i}} p\left(x^{\prime}\right)\right) \pi^{i}(y) .
$$

From relation 5.36 , we deduce expression 4.12 where the matrix $K$ is given by

$$
K_{i j}=\int_{Y_{f}} w_{j}^{i}(y) d y \quad i, j=1,2 .
$$

Let us now prove the Darcy equation 4.13$)$. To do this, we recall that $\hat{U}$ satisfies conditions $[5.46]_{3,4}$ and therefore, using identification $(5.49)$, we get

$$
\begin{aligned}
\sum_{j=1}^{2} \partial_{x_{j}}\left(\sum_{i=1}^{2}\left(f_{i}^{\prime}-\partial_{x_{i}} p\right) \int_{Y_{f}} w_{j}^{i} d y\right)=\operatorname{div}_{x^{\prime}} \int_{Y_{f}} \hat{U}^{\prime}\left(x^{\prime}, y\right) d y=0 & \text { in } \omega \\
\left(\sum_{i=1}^{2}\left(f_{i}^{\prime}-\partial_{x_{i}} p\right) \int_{Y_{f}} w_{j}^{i} d y\right) \cdot n=\left(\int_{Y_{f}} \hat{U}^{\prime}\left(x^{\prime}, y\right) d y\right) \cdot n=0 & \text { on } \partial \omega .
\end{aligned}
$$

On the other hand, we observe that taking $w^{i}$ as test function in the equation satisfied by $w^{j}$, we have

$$
\int_{Y_{f}} w_{j}^{i}(y) d y=\nu \int_{Y_{f}} D w^{i}: D w^{j} d y, \quad i, j=1,2,3 .
$$

Thus, 5.50 proves that $p$ satisfies the Darcy equation (4.13), which is an elliptic equation with $K$ a symmetric and positive definite matrix, see [13. Then problem 4.13) has a unique solution. Moreover, as $K f^{\prime} \in L^{2}(\Omega)$ and $Y_{f}$ is a subset of $Y$ which is smooth and connected, from regularity results for problem (see Chapter 7 in [12]) we conclude that $p \in H^{1}(\omega)$, which implies that $U$ (and so $\hat{U}$ ) is also unique. This proves that the entire sequence $\left(a_{\varepsilon}^{-2} \tilde{u}_{\varepsilon}, p_{\varepsilon}\right)$ converges to $(u, p)$. This finishes the proof. 


\section{References}

[1] G. Allaire, Homogenization of the Stokes flow in a connected porous medium. Asymp. Anal., 2 (1989) $203-222$.

[2] T. Arbogast, J. Douglas J.R. and U. Hornung, Derivation of the double porosity model of single phase flow via homogenization theory, SIAM J. Math. Anal., 21 (1990) 823-836.

[3] M. Anguiano, F.J. Suárez-Grau, Homogenization of an incompressible non-Newtonian flow through a thin porous medium, Z. Angew. Math. Phys. (2017) 68:45.

[4] M. Anguiano, F.J. Suárez-Grau, The Transition Between the Navier-Stokes Equations to the Darcy Equation in a Thin Porous Medium, Mediterr. J. Math. (2018) 15:45.

[5] G. Bayada, N. Benhaboucha, M. Chambat, Modeling of a thin film passing a thin porous medium, Asymptotic Analysis 37 (2004) 227-256.

[6] D. Cioranescu, A. Damlamian and G. Griso, Periodic unfolding and homogenization, C.R. Acad. Sci. Paris Ser. I, 335 (2002) 99-104.

[7] D. Cioranescu, A. Damlamian and G. Griso, The periodic unfolding method in homogenization, SIAM J. Math. Anal., 40 (2008) 1585-1620.

[8] P. G. Darcy, Les fontaines publiques de la ville de Dijon Paris. Victor Dalmont, 1856.

[9] J. Fabricius, J. Gunnar I. Hellström, T. Lundström, E. Miroshnikova and P. Wall, Darcy's Law for flow in a periodic thin porous medium confined between two parallel plates, Transp. Porous Med., 115 (2016) $473-493$.

[10] U. Hornung, Homogenization and Porous Media, Springer, New York, 1997.

[11] J.-L Lions, Some Methods in the Mathematical Analysis of Systems and Their Control. Science Press and Gordon and Breach, Beijing, 1981.

[12] E. Sanchez-Palencia, Nonhomogeneous media and vibration theory, Lecture Notes in Physics 127, SpringerVerlag, 1980.

[13] L. Tartar, Incompressible fluid flow in a porous medium convergence of the homogenization process. Appendix to Lecture Notes in Physics, 127, 1980.

[14] R. Temam, Navier-Stokes Equations, North Holland, 1984. 